\title{
Urgences
}

\section{B. Section intra du domaine}

\section{Renald Bérubé et André Gervais}

Numéro 19, janvier 1988

Le tour du texte

URI : https://id.erudit.org/iderudit/025443ar

DOI : https://doi.org/10.7202/025443ar

Aller au sommaire du numéro

Éditeur(s)

Urgences

ISSN

0226-9554 (imprimé)

1927-3924 (numérique)

Découvrir la revue

Citer ce document

Bérubé, R. \& Gervais, A. (1988). B. Section intra du domaine. Urgences, (19),

27-38. https://doi.org/10.7202/025443ar d'utilisation que vous pouvez consulter en ligne.

https://apropos.erudit.org/fr/usagers/politique-dutilisation/ 


\section{B. Section intra du domaine:}

textuation

intratexte, intratextualité

autotexte, autotextualité

entretexte

sous-texte

cotexte

microtexte: voir cotexte

onomatexte

épitexte

métatexte, métatextualité

non-texte: voir métatexte

pseudotexte: voir métatexte

métatextuel

textolecte

intertextolecte: voir textolecte

biotexte

surtexte

milletexte (mixte) 


\section{Textuation}

\section{RÉF. - Michel van Schendel, 1986-1987}

DÉF. — «l... comment justifier l'appellation de «PRATIQUE SÉMIOTIQUE» réservée par Kristeva au «texte»? La textuation en est vraisemblablement une, à savoir l'acte d'écriture ou de lecture qui reproduit, recompose et/ou transforme fragments et formes discursives en un ensemble appelé récit, poème, article, etc. L'étude de cet acte relève d'une pragmatique. Et l'examen d'un échantillon de ces actes devrait révéler, non seulement des frontières nouvelles entre genres reconnus, voire des déplacements de genres, mais au-delà et à l'inverse lemploi de formes et procédures communes entre agents écrivants, écrivains, conteurs, lecteurs, auditeurs, parlants, parolants et paroliers, écouteurs et inventeurs (je me permets cette remarque partiellement néologisante, pour indiquer des extensions encore inanalysées): On aurait alors, hypothétiquement, les premières indications d'une analyse non réductive portant sur la pratique sociale homogène de ces agents dappareils spécialisés, et sur les différences sémiotiques manifestées dans l'exercice de cette pratique. A ce compte, on peut parler de pratique sémiotique. À ce compte seulement.

Mais Kristeva ne parle pas de textuation, bien qu elle prononce les mots "procès" et "pratique». Son «texte», seulement scriptural, est isolé d'une "énonciation" elle-même rabattue sur la disjonction/ conjonction des «énoncés» identifiés à une "parole» devenue effet de langue. Ce "texte" ainsi isolé est un démiurge. L'énonciation n'y est pas, la parole non plus. Quels sont les ressorts de ce qui, dans la parole et dans l'énonciation, n'est pas aussi textuel? Quel est donc cet acte, quels moyens a-t-on de le décrire, et quels en sont les liens avec sa pratique sociale si l'on veut parler de "pratique"? La réponse est d'autant plus mystérieuse que le texte semble être opposé à luimême. Plus exactement, il y aurait deux sortes de textes, opposées l'une à l'autre, bien que la notion de "texte" qui leur est commune ne ressorte pas de cette opposition. Il y aurait le texte ouvert de la "coupure épistémologique» opérée au XIXe siècle. Et il y aurait le «texte clos» fermé sur sa "parole», c'est-à-dire sur l'«idéologème du signe».

En conjoncture, dans la situation $N$ sociale de l'interdiscours inhérente à $l^{* *}$ intertexte, des temporalités courtes interfèrent avec les temporalités longues des modes de lecture, les unes et les autres étant également sectorielles. Il s'agit d'abord de la textuation proprement dite, d'une sorte de phénoménologie de l'acte singulier d'écriture, ou plutôt d'un acte-phénomène corrigé par les décisions secto- 
rielles d'appareils. Les lecteurs appointés des maisons d'édition le laissent entendre couramment: On n'écrit plus comme ça aujourd'hui: ou encore: On n'écrit pas comme ça (aujourd'hui, pas encore); ou même: On n'écrit pas comme ça (vous commettez des fautes de style et de narration). Ces énoncés quasi préceptuels, qui insistent sur l'uniformité du «On», sont les indices de normes exécutées par les appareils d'édition et de mise en marché du livre. Mais ils sont aussi, en concomitance, les marques censurantes d'un rappel des actes singuliers d'écriture. "On n'écrit pas ainsi aujourd'hui." Qu'est-ce que cela signifie? Dans la textuation, la temporalité historique des modes d'écriture est plus "courte», plus immédiate que celle des modes de lecture qui lui sont liés. L'acte d'écriture, dans son secteur relatif, est homologue à l'acte de l'exécution politique."

Michel van Schendel: "L'idéologème est un quasi-argument». Texte. Toronto, no 5/6 (no intitulé Théories du texte), 1986-1987, p. 98 et 117.

\section{Intratexte, intratextualité}

RÉF. - ?

DÉF. — «S'agissant de (mécanismes du) texte, il y a, on le sait, des précédents: intratexte, à savoir la relation que le texte entretient avec lui-même à compter d’éléments déterminés, et *intertexte, à savoir une "relation construite" [J. Ricardou) entre deux textes."

Andre Gervais, Jean-Pierre Vidal et Ghislain Bourque: "Propositions initiales». La nouvelle barre du jour, Montréal, no 103 (no intitulé Linfratexte), mai 1981, p. 9.

>> L'intratexte est donc le texte lorsqu il est pris dans une relation duelle: intratexte $/{ }^{*}$ intertexte, intratexte $/{ }^{* \prime}$ extratexte.

\section{Autotexte, autotextualité}

RÉF. — Lucien Dällenbach, 1976

DÉF. - «Dans l'étude présentée au colloque Claude Simon de Cerisy-la-Salle (1974), une discrimination est établie [par J. Ricardou] entre *intertextualité générale (rapports intertextuels entre textes d'auteurs différents) et * intertextualité restreinte (rapports intertextuels entre textes du même auteur). [... II faudrait) reconnaitre l'existence, à côté de l'* intertextualité générale et de l'* intertextualité restreinte, d'une * intertextualité autarcique. Afin de mettre l'accent sur son originalité propre et de ne pas heurter par un nouveau 
prédicat des habitudes lexicales bien ancrées, nous proposons, dans le sillage de Gérard Genette, de nommer cette * intertextualité autarcique autotextualité."

Lucien Dällenbach: «Intertexte et autotexte». Poétique. Paris. no 27, 1976, p. 282.

EX - Cet article n'analyse que le premier des 3 autotextes quil mentionne: mise en abyme (ou résumé), auto-citation, variante.

\section{Entretexte}

RÉF. - Jacques Allard, 1987

DÉF. - «[...) une formation romanesque qu'il faudrait appeler un "entretexte», pratique systématique de l'intermittence narrative, thématique, énonciative; mise en relief de l'intervalle spatio-temporel; prédominance de l'intermédiaire actoriel... pour un théâtre de l'entracte et une poétique de l'entrefaite».

Jacques Allard: «Avant-texte pour demain: Prochain épisode d'Hubert Aquin", Littérature, Paris, no 66 (no intitulé Recherches québécoises), mai 1987, p. 87.

EX. - L'article ici cité, surtout p. 84-90.

\section{Sous-texte}

RÉF. - Pierre van den Hęuvel, 1985

DÉF. - "Comme la conversation anodine, le discours textuel se désiste de sa fonction informationnelle (le récit) pour n'être qu'un lien sonore, établi par l'allocution, par les «tropes" de voix imitées qui profèrent des paroles dénuées de sens, un bégaiement, une «jactance». Comme la sous-conversation affective, le texte peut contenir un "sous-texte» qui, comme une ombre, introduit l'inconscient entre les mots et, comme une voix off, procure au discours une certaine musicalité et une énergie toute particulière.»

Pierre van den Heuvel: Parole mot silence. Pour une poétique de lénonciation, Paris, Corti, 1985, p. 62.

>> Fait, comme l'indique une note de la même page, sur "sousconversation" - voir Nathalie Sarraute: "Conversation et sousconversation", dans L'ère du soupçon (1956) - , ce terme semble bien au carrefour de quelques autres: de l'* infratexte d'une part, de 


\section{Cotexte}

RÉF. — Janos S. Petöfi, 1971 - Transformationsgrammatiken und eine ko-textuelle Textheorie. Grundfragen und Konzeptionen, Frankfurt am Main, Athenäum, 1971

DÉF. - "Nous considérons le titre comme un texte, souvent déformé, peu grammatical et fort condensé, mais parfois aussi parfaitement régulier, se composant d'une phrase complète (Les lauriers sont coupés, Dujardin) ou, rarement, d'une série de phrases enchaînées. Le titre n'est donc pas vu comme une partie intégrante du co-texte (...). NOTE: Le terme «co-texte» est emprunté à Petöfi (1971) pour indiquer ici l'ensemble de phrases qui suivent ou qui devraient suivre le(s) titre(s) mentionné(s) à la page de titre. Le co-texte est ainsi léquivalent du texte dépourvu de son titre. [...]

Placé en tête du texte, au milieu d'un blanc, isolé du co-texte sur une page spéciale et écrit en majuscules, le titre saffirme comme dirigeant du co-texte: il programme d'avance la lecture et impose au lecteur la valeur de son énoncé."

Leo H. Hoek: La marque du titre. Dispositifs sémiotiques d une pratique textuelle. La Haye, Paris, New York. Mouton, 1981, p. 17-18 et 283.

>> Chez Petöfi et chez Hoek, le titre est au texte (ou cotexte) ce que, chez Jacques Duchet (" "La fille abandonnée" et "La bête humaine". Éléments de titrologie romanesque", Littérature, Paris, no 12 (no intitulé Codes littéraires et codes sociaux), décembre 1973, p. 51), le titre (ou microtexte) est au texte. Faut-il ajouter que le terme de "microlecture" i décrivant le type de lecture (petite lecture) et d'objet lu (lecture du petit); terme proposé par Jean-Pierre Richard dans les deux tomes déses Microlectures (Paris, Seuil, coll. «Poétique», 1979 êt 1984), na pás de rappoit, sinon très ponctuellement, avec le terme ici proposé par J. Duchet. Mais il pourrait advenir, qui sait, que l'objet "d'une microlecture (pas nécessairement richardienne) soit un microtexte (et paś nécessairement un titre).

EX. - Ada M. Vilar de Kerkhoff: "Écho et Narcisse: réflexions et réfractions du rapport titre-texte sur l'espace de la traduction». Texte, Toronto, no 4, 1985, p. 127-135. 


\section{Onomatexte}

RÉF. - Jean Ricardou, 1978

DÉF. - «Ni autre texte, ni mème texte, le titre est un onomatexte: il forme le nom du texte. Or, on distingue deux types de noms: celui qui est offert par la langue, celui qui est proposé par tel de ses utilisateurs. Dans la mesure où le texte est un objet nouveau, nul terme de la langue qui à première vue lui convienne: le titre relève de la néologie." Jean Ricardou: Nouveaux problèmes du roman. Coll. «Poétique», Paris. Seuil, 1978. p. 143.

EX. - Les p. 145-150 du livre ici cité proposent une analyse d'une quinzaine de titres.

\section{Épitexte}

RÉF, - Jean Ricardou, 1978;'Gérard Genette, 1987

DÉF. - "Titre, résumé, définition: ainsi s'élargit l'ensemble qui échappe à la fois aux règles de $\mathrm{l}^{\prime *}$ intertexte et de l'* intratexte. Non moins que le titre, le résumé et la définition sont en même temps ce qui se distingue et ce qui n'a pas d'autonomie. lls appartiennent à ce qu'il faudrait nommer l'épitexte: le texte sur le texte. Le * métatexte est écrit sur le texte à des fins opératoires, analytiques; l'épitexte est écrit sur le texte à des fins représentatives, synthétiques. L'épitexte appartient donc à un continent plus vaste: le synonymique, auquel la pensée au pouvoir réserve les seules substitutions licites."

Jean Ricardou: Nouveaux problèmes du roman. Coll. «Poétique», Paris, Seuil, 1978, p. 144.

$\S$ «Autour du texte encore, mais à distance plus respectueuse (ou plus prudente), tous les messages qui se situent, au moins à l'origine, à l'extérieur du livre: généralement sur un support médiatique (interviews, entretiens), ou sous le couvert d'une communication privée (correspondances, journaux intimes, et autres). C'est cette deuxième catégorie que je baptise, faute de mieux, épitexte, et qui occupera les deux derniers chapitres. [...]

Ce qui distingue l'épitexte privé de l'épitexte public n'est pas exactement labsence de visée du public, et donc d'intention de publication: bien des lettres, bien des pages de journal sont écrites dans une claire prescience de leur publication à venir, et l'effet qu exerce sans doute cette prescience sur leur rédaction nentame 
pas leur caractère privé, voire intime. Ce qui définira pour nous ce caractère, c'est la présence interposée, entre l'auteur et l'éventuel public, d'un destinataire premier (un correspondant, un confident, l'auteur lui-même) qui n'est pas perçu comme un simple médiateur ou relais fonctionnellement transparent, une «non-personne» médiatique, mais bien comme un destinataire à part entière, à qui l'auteur s'adresse pour lui-même, fût-ce avec l'arrière-pensée de prendre ultérieurement le public à témoin de cette interlocution. Dans l'épitexte public, l'auteur s'adresse au public, éventuellement à travers un médiateur, dans l'épitexte privé il s'adresse d'abord à un confident réel, perçu comme tel, et dont la personnalité importe à cette communication, jusqu'à en infléchir la forme et la teneur.»

Gérard Genette: Seuils. Coll. «Poétique», Paris, Seuil, 1987, p. 10-11 et 341.

EX. - Lesdits derniers chapitres de Seuils (p. 316-370).

\section{Métatexte, métatextualité}

RÉF. - ? (sur le modèle de métaphysique); Jean Ricardou, 1978

DEFF. — «Par nature, les faits que relate la mythologie n'existent qu'en tant qu'ils sont dits. Ils sont avant tout des êtres de langage, et pourtant leur rapport avec le langage est profondément original.

Si l'on veut se faire de la mythologie une image qui ne soit pas figée, il faut affirmer qu'un mythe n'est pas réductible à la somme des oeuvres qui en traitent. S'il l'était, il ne serait que littérature. Pour être un mythe, il doit posséder en outre, au moins virtuellement, un domaine non verbal. Le paradoxe est que ce domaine est pourtant jalonné de textes, dont les uns existent et les autres, nouveaux ou à créer, sont suscités par la vie propre du mythe. (...)

[...] De même que la physique d'Aristote se prolonge par une méta-physique, on pourra désigner par le terme de méta-texte la réalite en quelque sorte trans-verbale de la mythologie. Elle contient des mots et nous atteint par des mots, mais elle est aussi autre chose.

S'il a pu devenir un méta-texte, c'est parce que l'élément mythologique était d'abord un non-texte. Un texte ordinaire, somme de mots, n'aurait aucune raison de se transmuer en un méta-texte doué d'autres vertus. La mobilité, semblable à celle de la vie, de la réalité mythologique fait d'elle à la fóis un dit et un non-dit. D'une part en effet le mythe s'exprime selon des dimensions très particulières, qui ne sont pas celles de la littérature, d'autre part la vacance qui est en lui est toujours une réserve potentielle de créations nouvelles.

[...] Le mythe n'a, grâces en soient rendues à nos ignorances 
historiques, pas d'auteur. Il n'est pas inscrit dans le temps, ce qui lui permet d'être aussi actuel aujourd'hui que lors de la guerre de Troie.

Il présente pourtant, tout comme un texte, une fable, ou mème plusieurs; celles-ci peuvent être successives ou simultanées, offrant dans ce dernier cas, soit des contradictions, soit un sens plus riche et plus complexe qu'un récit linéaire. Aussi bien le sens du mythe n'est-il pas plus fixé que son texte: d'autres interprétations, ou d'autres générations, y verront d'autres valeurs.

Si beaucoup de ces caractères sont négatifs, c'est que le mythe, en même temps que méta-texte, est un pseudo-texte. Il est intrinsèquement fiction et c'est pourquoi, plus que calque dư réel, il est oeuvre d'art. (...)

On peut encore le comparer à ces dessins dont les esquisses et les propositions non retenues n'ont pas été effacées et continuent de jouer à côté du tracé qui serait, dans une perspective plus étroite, la seule et exclusive vérité. Le méta-texte est pluriel. Il est l'antithèse de cette curieuse activité, née au XIXe siècle et baptisée "établissement du texte". Il s'agissait de relever, d'étudier et de classer toutes les variantes, lacunes, interpolations ou absurdités des manuscrits plus ou moins fautifs d'un même texte, de comparer également, sil y en avait, les variations de toutes les éditions existantes, pour en tirer par des règles complexes, un texte unique, déclaré le seul bon et vrai. $\grave{A}$ l'opposé, les considérations de la mythologie comme méta-texte amènent à ne plus s'offusquer de l'apparent paradoxe de Valéry qui, étendant au texte poétique la labilité du méta-texte, affirmait qu'il n'y a pas de vrai sens d'un texte. Le mythologue verrait plutôt dans cette proposition une évidence."

Jacques Scherer: Dramaturgies d'Oedipe, Paris. PUF, coll. «Écriture», 1987, p. $143-$ 146.

>> Les termes de non-texte et de pseudotexte appartenant en propre à cette définition de J. Scherer désignent la part de réalité trans-verbale (non textuelle) du mythe et l'apport de chaque version (textualisée) au mythe. Le mythe seul, ici, objet privilégié de ce livre, autorise une si large ouverture qui mène du non-textuel au textualisé.

>> De façon plus restreinte, $M$. Angenot (Glossaire pratique de la critique contemporaine, p. 131) appelle métatexte le sens allégorique ("second sens qui est sous-jacent au sens littéral») que peut avoir, par exemple, un conte.

$\S$ «Le métatexte est écrit sur le texte à des fins opératoires, analytiques [...].»

Jean Ricardou: Nouveaux problèmes du roman. Coll. «Poétique», Paris, Seuil, 1978. p. 144. 
+ "Le troisième type de transcendance textuelle [* transtextualité], que je nomme métatextualité, est la relation, on dit plus couramment de "commentaire", qui unit un texte à un autre texte dont il parle, sans nécessairement le citer (le convoquer), voire, à la limite, sans le nommer [...]. C'est, par excellence, la relation critique. On a, naturellement, beaucoup étudié (méta-métatexte) certains métatextes critiques, et l'histoire de la critique comme genre; mais je ne suis pas sûr que l'on ait considéré avec toute l'attention qu'il mérite le fait même et le statut de la relation métatextuelle."

Gérard Genette: Palimpsestes. La littérature au second degré. Coll, «Poétique», Paris, Seuil, 1982, p. 10.

+ «Reste que la dimension critique et théorique de la préface allographe l'entraîne manifestement vers la frontière qui sépare (ou plutôt vers l'absence de frontière qui ne sépare pas nettement) le * paratexte du métatexte, et plus concrètement la préface de l'essai critique.»

Gérard Genette: Seuils. Coll. «Poétique», Paris, Seuil, 1987, p. 249.

>> Les deux acceptions de ce terme, fort éloignées l'une de l'autre, utilisent deux connotations du préfixe «méta-»: au-delà et sur. Le terme, dans la première acception, s'occupe du mythe (sa part de réalité trans-verbale), dans la seconde, du texte littéraire en tant que tel.

\section{Métatextuel}

RÉF. - Bernard Magné, 1980 - «Le métatextuel». Actes du colloque d'Albi: langages et signification, Toulouse, Université de Toulouse-Le-Mirail, 1980, p. 228-260.

DÉF. — «[...] de quels moyens un texte dispose-t-il pour assurer dans son corps mêrme la désignation de tout ou partie de ses mécanismes constitutifs?

L'ensemble de ces moyens forme ce que j'ai nommé ailleurs et depuis déjà un certain temps le métatextuel. [...]

Relève du métatextuel dénotatif tout énoncé dont les unités linguistiques véhiculent des informations implicites sur un référent langagier dont cet énoncé constitue tout ou partie. [...]

Dans le métatextuel connotatif, le signifié de connotation est toujours spécifique puisque les informations qu'il fournit portent sur autre chose que le référent du discours. En effet, le référent du discours, c'est, en ce cas, la "fiction", l'univers diégétique, alors que 
les informations métatextuelles concernent les procédés formels produisant cette fiction, cet univers.»

Bernard Magné: «Métatextuel et lisibilité». Protée. Chicoutimi, vol. 14, no 1-2 (no intitulé La lisibilité), printemps-été 1986, p. 77,78 et 80.

>> On aura noté que ce terme, adjectif de ${ }^{*}$ métatexte, est ici, selon le sens donné par B. Magné, un substantif à part entière. D'où la nécessité de lui consacrer une entrée distincte.

>> > On ajoutera qu'en au moins une de ses avancées ( «l'essentiel demeure ce mécanisme par lequel le texte produit ses garanties à l'égard du code en se métatextualisant "), l'article de Jacques Dubois ("Code, texte, métatexte», Littérature, Paris, no 12 (no intitulé Codes littéraires et codes sociaux), décembre 1973, p. 9) s'approche de la définition que B. Magné donnera du métatextuel.

EX. - L'article ici cité ("Métatextuel et lisibilité») analyse plusieurs fragments de G. Perec et J. Ricardou, notamment.

\section{Textolecte}

RÉF. — Jean Ricardou, 1978

DÉF. - «En la rigueur de ses mécanismes, le texte tend en effet, si peu qu'on y prenne garde, à construire son propre vocabulaire et peut-être davantage: ce qu'on pourrait nommer un texto-lecte. L'un des principes de cette élaboration lexicale spécifique revient à établir entre deux termes très distincts une solidarité apte à produire, aberrante hors le texte, une manière de synonymie textuelle. [...]

Tantôt, il peut s'agir d'un rapprochement de termes dont la solidarité spécifique est obtenue implicitement par un effet de redites calculées. (...)

Tantôt, il peut s'agir d'un rapprochement de termes dont la solidarité spécifique est obtenue explicitement par un effet de commentaire concerté."

Jean Ricardou: Nouveaux problèmes du roman. Coll. «Poétique», Paris, Seuil, 1978, p. 114 et 288.

>> > Le mot intertextolecte n'est actualisé (Nouveaux problèmes du roman, p. 296) que sous sa forme adjectivale (intertextolectal). 


\section{Biotexte}

\section{RÉF. - Jean Ricardou, 1982}

DÉF. - «Avec la biographie, il s'agit de fournir, au cours de tel écrit, les notables événements qui marquent le fil d'une existence. Avec le biotexte, il s'agit de choisir, au coeur de telle vie, les précis éléments qui obéissent à certaines règles du texte en fabrique. [...] Avec la biographie, du moins en apparence, les événements s'imposent censément au geste qui inscrit. Ou, si l'on préfère, le fonctionnement est de l'ordre de l'expression ou de la représentation. Avec le biotexte, les éléments sont requis par l'acte de l'écriture. Ou, si l'on aime mieux, le mécanisme est du registre de la * textualisation.»

Jean Ricardou: Le théâtre des métamorphoses. Coll. "Fiction $\varepsilon$ Cie», Paris, Seuil, 1982, p. 188.

EX._Le livre ici cité: le passage intitulé «Postface»(p. 186-196, còté pair seulement).

\section{Surtexte}

RÉF. - Jean Ricardou, 1978

DÉF. - «[..] le rapprochement de deux passages disjoints du texte sera accompli, non plus seulement par la lecture, mais aussi par l'écriture elle-même. Telle inscription scandaleuse, nous le verrons, de la translinéarité (rapprochement exclusivement lectural) dans la linéarité (rapprochement devenant scriptural) ouvre sur un autre domaine: le sur-translinéaire ou, pour être bref, le surtexte. Le surtexte advient ainsi à chaque fois que le texte s'élabore en écrivant une lecture de lui-même. On le conçoit: cette inscription du translinéaire dans le linéaire peut se réussir au moins de deux manières: l'une, directe; l'autre, indirecte. Dans le premier cas, l'interruption du translinéaire se dispose sans médiation: ce qui s'écrit à tel endroit du texte, ce n est plus une invitation implicite faite à la lecture de se reporter à tels autres passages du texte, c'est l'obligation explicite faite à la lecture de se reporter à tel autre passage du texte parce que cet autre passage du texte se trouve également écrit mot pour mot ici même. Avec le surtexte, ce qui se propose, c'est une façon d'ubiquité du texte: tel passage se trouve en quelque sorte textuellement multiplié et réparti, tel quel, en divers lieux du texte. (...)

Dans (le second) cas, l'irruption du translinéaire se dispose selon une médiation: celle d'une théorie. Ce qui s'écrit à tel endroit du texte, ce 
n'est plus une invitation implicite faite à la lecture de se reporter à tel autre passage du texte, c'est l'obligation faite à la lecture de se reporter à tel autre passage du texte parce que cet autre passage du texte se trouve également écrit mot pour mot ici même, dans le cadre dune réflexion sur le texte."

Jean Ricardou: Nouveaux problèmes du roman. Coll. "Poétique», Paris, Seuil. 1978, p. $272-273$ et 277 .

>> L'inscription indirecte du surtexte fait advenir, au coeur du texte, ce que $B$. Magné nommera le * métatextuel.

\section{Milletexte [Mixte]}

RÉF. - Jean Ricardou, 1982

DÉF. — «Que l'on suppose un texte établi selon le multiple mais qui se refuse au nom hyperbolique de "milletexte» (...). Prime temps: il s'impose que le terme à soustraire (milletexte) soit tributaire d'une ferme mise en cause. Si l'on pouvait lui opposer une tapisserie, son enflure, par discrète anagramme, deviendrait matière de pâtisserie. Si l'on pouvait l'assimiler à une pâtisserie, son allure, par discrète homogramme, se ferait manière de "millefeuille». Second temps: il importe que le couple de mots solidaires (mille-texte) se comporte en un souple mot solitaire. Tiers temps: il convient que le terme sommaire nullement ne se ferme au jeu multipolaire:

La tapisserie, ainsi offerte, il est temps, en la réduction de toute emphase littérale, se nommera un mixte.

Quatre temps: il devient élémentaire que l'impudique diversité (le milletexte) se restreint en laconique variété (le mixte), avec, d'un trait réussi, le précis retrait d'un segment (llete) qui, justement, en son anagramme (telle), y maintenant l'oriflamme du similaire.» Jean Ricardou: Le théâtre des métamorphoses. Coll. «Fiction $\mathcal{E}$ Cie», Paris, Seuil, 1982, p. 77.

EX. - Le livre ici cité est dit «mixte» comme tel autre livre est dit «roman» ou «poèmes», par exemple. 